\title{
Letter to the Editor: Optimal Prediction of the Central Venous Catheter Insertion Depth Targeting the Cavoatrial Junction
}

\author{
U. S. Dadhwal ${ }^{1}$
}

Accepted: 21 June 2020/Published online: 20 July 2020

(C) Société Internationale de Chirurgie 2020

\section{Dear Editor,}

I read with interest Hyejin's paper on Optimal Prediction of the Central Venous Catheter length [1].

The author has greatly simplified the method of prediction of an accurate length of CVC to be inserted so as to place its tip near cavo-atrial junction.

In their conclusion, they have highlighted that most of the CVC's are inserted at the bedside and their formula provides a handy and practical guidance. The formula reads as $\mathrm{L} 1+\mathrm{L} 2+4.4 \mathrm{~cm}$.

To further simplify this calculation, I would suggest the following:

L1-From point of needle insertion to suprasternal notch/jugular notch (not the clavicular notch as suggested by the authors).

L2-From suprasternal notch to sternal angle or angle of Louis, as this bony ridge is palpable under the skin and a chest X-ray is not required for its identification. In many situations, radiology services may not be available either immediately or the patient's medical condition may not permit to shift for a chest radiograph. It is easier to identify the sternal angle by simple palpation than to identify the carina on chest radiology. In certain pulmonary conditions, the carina may not be easily identifiable on imaging, leading to wastage of precious time.

As we understand that the carina/tracheal bifurcation lies either at the level of sternal angle [2] or slightly below it [3], the alpha can be recalculated from sternal angle to

U. S. Dadhwal

usd999@rediffmail.com

1 B1, 402, Nirmal Chaya Towers, VIP Rd, Zirakpur, Punjab, India cavo-atrial junction using the IV contrast method as mentioned in the article. The sternal angle can be identified on chest radiograph by sticking a radio-opaque marker on skin to calculate distance from cavo-atrial junction. The new formula will obviate the need for a chest $\mathrm{X}$-ray in real emergency situations and make it an easy bedside procedure.

I hope that the author will kindly recalculate the alpha distance and provide us with the formula as it is certainly of great use in emergency situations.

Compliance with ethical standards

Conflict of interest The author declares that he has no conflict of interest.

\section{Reference}

1. Mo H, Ahn S, Lee J, Cho S, Min SK (2020) Optimal prediction of the central venous catheter insertion depth targeting the cavoatrial junction. World J Surg 44(7):2170-2174. https://doi.org/10.1007/ s00268-020-05449-7

2. Spratt JD, Tunstall R (2016) Section 7, Thorax: Chapter 51 overview and surface anatomy. In: Gray's anatomy: the anatomical basis of clinical practice, Elsevier Limited, Amsterdam, p. 898

3. Mirjalili S, Hale S, Buckenham T et al (2012) A reappraisal of adult thoracic surface anatomy. Clin Anat 25:827-34

Publisher's Note Springer Nature remains neutral with regard to jurisdictional claims in published maps and institutional affiliations. 